\title{
The Conway-Kochen Argument and relativistic GRW Models
}

\author{
Angelo Bass:* \\ Mathematisches Institut der Universität München, \\ Theresienstr. 39, 80333 München, Germany, \\ The Abdus Salam International Centre for Theoretical Physics, Strada Costiera 11, 34014 Trieste, Italy. \\ GianCarlo Ghirardi甲 \\ Department of Theoretical Physics and I.N.F.N., Strada Costiera 11, 34014 Trieste, Italy, \\ The Abdus Salam International Centre for Theoretical Physics, Strada Costiera 11, 34014 Trieste, Italy.
}

\begin{abstract}
In a recent paper, Conway and Kochen proposed what is now known as the "Free Will theorem" which, among other things, should prove the impossibility of combining GRW models with special relativity, i.e., of formulating relativistically invariant models of spontaneous wavefunction collapse. Since their argument basically amounts to a non-locality proof for any theory aiming at reproducing quantum correlations, and since it was clear since very a long time that any relativistic collapse model must be non-local in some way, we discuss why the theorem of Conway and Kochen does not affect the program of formulating relativistic GRW models.
\end{abstract}

KEY WORDS: Free Will theorem, entangled states, non-locality, collapse models.

\section{THE CONWAY-KOCHEN ARGUMENT}

We briefly review the argument by Conway and Kochen [1]. Following their way of presenting it, we do not assume any particular theory underlying physical phenomena; we only assume that certain particular physical systems exist — independently of how they are described by any theoryupon which measurements can be made; more specifically, we assume that there exist systems which we call "particles of spin 1", upon which operations which we call "measurement of the square of the spin along the direction $n$ " can be performed ( $n$ denotes any direction in the three-dimensional

*Electronic address: bassi@mathematik.uni-muenchen.de

${ }^{\dagger}$ Electronic address: ghirardi@ts.infn.it 
space); we call $S_{n}^{2}$ the outcome of such a kind of measurement along the direction $n$. We assume that when $x, y, z$ represent an orthogonal triple of directions, the three corresponding measurementoperations can be simultaneously performed on the system ${ }^{1}$; we assume furthermore, in agreement with Quantum Mechanical rules, that:

$$
\begin{aligned}
& S_{n}^{2} \text { takes only the value } 0 \text { or } 1 \text {, for any } n \text {; } \\
& \text { For any orthogonal triple } x, y, z \text {, one has: } S_{x}^{2}+S_{y}^{2}+S_{z}^{2}=2 \text {, }
\end{aligned}
$$

whenever the three corresponding measurements are (simultaneously) performed. Trivially, properties (11) imply that one of $S_{x}^{2}, S_{y}^{2}, S_{z}^{2}$ is 0 , while two are 1 .

We finally assume the standard formalism of special relativity, in particular concepts like the backward light-cone and space-like separated regions of spacetime.

\subsection{The Postulates}

Conway and Kochen consider the following three axioms:

TWIN: It is possible to produce two spin 1 particles which are in the state of "total spin 0", meaning with this that if a measurement of the square of the spin along the direction $n$ is performed on one particle, giving the outcome $S_{n}^{2}$, then a measurement of the square of the spin along the same direction $n$ performed on the other particle gives the same outcome $S_{n}^{2}$. Moreover, such a property does not depend on the relative position of the two particles and on the relative time on which the two experiments are performed; in particular, it holds true when the two measurements are space-like separated.

FREE: Each experimenter can freely choose any direction $n$ along which to perform the measurement.

FIN: Information cannot travel at a speed greater than the speed of light.

In the last axiom, we used the term "information" in an intuitive sense, without specifying what it means; though we do not like to resort to such a vague term in setting the axioms of any logical reasoning, we use it simply to adhere to the original formulation of [1].

\footnotetext{
${ }^{1}$ This property reflects the well known fact that, in the case of a spin 1 particle, the squares of the spin operators along three orthogonal directions commute among themselves.
} 


\subsection{The Argument}

Let us consider two spin 1 particles which are in a state of total spin 0 ; let us label with " $a$ " one of the two particles and with " $b$ " the other one, and with " $A$ " an experimenter who performs a measurement on $a$, and with " $B$ " one who performs a measurement on $b$. Let $n, m$ and $\ell$ be three orthogonal axis along which $A$ decides to perform three practically simultaneous measurements of the square of the spin of particle $a$.

We assume that the outcome of the measurement of the square of the spin of particle $a$ along the direction $n$ is a function of all the information $\alpha$ contained in the backward light-cone of the particle (with respect to the spacetime point where the measurement is performed), and of the other two directions ${ }^{2} m$ and $\ell$ chosen by $A$ :

$$
S_{a: n}^{2} \equiv S_{a: n}^{2}(m, \ell ; \alpha)
$$

(we have slightly changed the notation from $S_{n}^{2}$ to $S_{a: n}^{2}$ in order to distinguish when $S_{n}^{2}$ refers to a measurement performed on particle $a$ and when it refers to a measurement performed on particle $b$, along the direction $n$ ).

In a similar way, if $B$ decides to simultaneously measure the square of the spin of particle $b$ along three orthogonal directions $i, j, k$, we assume that the outcome $S_{i}^{2}$ may depend only on the information $\beta$ contained in the backward light-cone and on the other two directions $j, k$ chosen by $B:$

$$
S_{b: i}^{2} \equiv S_{b: i}^{2}(j, k ; \beta)
$$

Assume finally that the two experimenters perform their measurements at space-like separated regions, so that there cannot be any exchange of information among them before the two measurements are over. The argument now goes as follows.

Because of TWIN, if $A$ and $B$ choose a common axis $n$, then the two outcomes must be perfectly correlated:

$$
S_{a: n}^{2}(m, \ell ; \alpha)=S_{b: n}^{2}(j, k ; \beta)
$$

for any direction $n$. But, because of FREE, $B$ can choose to perform his measurement along any orthogonal triple $n, k, j$, with $n$ fixed. Because of FIN, such a choice cannot affect $S_{a: n}^{2}(m, \ell ; \alpha)$.

\footnotetext{
${ }^{2}$ The necessity of allowing, in principle, that the outcome along $n$ depends on the other chosen directions follows from the so-called Kochen-Specker theorem [2].
} 
This means that the outcome of the spin measurement along $n$ cannot depend on which other two orthogonal directions $j, k$ are chosen by the experimenter, i.e. :

$$
S_{b: n}^{2}(j, k ; \beta)=S_{b: n}^{2}(\beta)
$$

Obviously, a similar conclusion holds for $S_{a: n}^{2}$.

The contradiction now arises because, as it is well known, a function like $S_{a: n}^{2}(\alpha)$ or $S_{b: n}^{2}(\beta)$ cannot exists [2]. As a consequence, the outcome $S_{a: n}^{2}$ of the measurement of the square of the spin of particle $a$ (or $b$ ) cannot be uniquely determined by the past information contained in the backward light-cone from the region where A (or B) performs its measurement.

\subsection{Comments}

The provocative conclusion of Conway and Kochen is that the particles' response to the experiment is free, i.e. that the outcome of an experiment cannot be entirely determined by the previous information accessible to whom performs the measurement, but indeed the moral stemming out of the above argument is well known and less surprising: no local theory exists which fully agrees with quantum mechanical predictions. As a matter of fact, what Conway and Kochen have shown is that the three postulates listed above, plus the existence of a functional relation between the outcomes of certain spin experiments and certain "information", lead to a contradiction, from which they conclude that such a functional relation cannot exist. But indeed, after the work of Bell it is well known that the conclusion is a different one: Nature is non-local, i.e. FIN is wrong, if, as the authors seem to suggest, information - apart from its ambiguous meaning-includes everything which might possibly determine an event (in our case, the outcome of a certain experimental procedure). And indeed, the above argument was first proposed by P. Heywood and M.L.G. Redhead 3] and by A. Stairs [4], further explored by H.R. Brown and G. Svetlichny [5] and subsequently generalized by A. Elby [6] (see also [13]); in these papers the above theorem is correctly presented as a non-locality proof, its novelty being that it differs from the original proof by Bell, by combining ideas previously related only to contextuality ${ }^{3}$.

The reason why after Bell's work one has to conclude that Nature is non-local is the following: Bell's theorem does not require the existence of a functional relation between the outcomes of experiments and past information. It simply requires the FREE assumption, the analog of the TWIN axiom for 1/2-spin particles, and Bell's definition of locality, which in some sense is the

\footnotetext{
${ }^{3}$ We thank S.L. Adler and D. Dürr for having brought the above papers to our attention.
} 
analog of the FIN axiom, even though it is expressed in clearer mathematical and physical terms. From these three axioms - we insist: without assuming any other functional dependence - one can derive an inequality which turns out to be violated by Nature. Accordingly, one of the three axioms must be wrong. Since TWIN has been experimentally verified and no-one is willing to deny FREE, then Bell's locality must be violated: Nature, in a very precise sense, is non-local.

Here we do not want to discuss the merits of the different proofs, whether Bell's definition of locality is more or less general than those which have been subsequently proposed, included the above FIN axiom; the moral is basically the same as the one given by Bell's theorem: any theory, in one way or another, must be non-local if it has to be empirically equivalent to Quantum Mechanics.

We stress it once more: the series of experiments performed by A. Aspect [7, 8] proved that Nature, by violating Bell's inequalities, violates Bell's condition of locality: this means, as far as we understand physics now, that Nature is non-local, that something happening in some region of space can affect the state of far away systems. On the other hand, as proven in [9, 10] for a quantum theory with the reduction postulate, such non-locality cannot be used to signal at a speed greater than the speed of light; this is what has been called the "peaceful coexistence" between Quantum Mechanics and Special Relativity [11], which renders Quantum Mechanics compatible with Special Relativity in spite of its non-local character.

People working on collapse models were of course aware of this non-locality constraint which any collapse model, whether relativistic or not, has to obey in order to be compatible with Quantum Mechanics; thus, for them, the argument of Conway and Kochen does not come as a surprise.

But there is something more. In applying their theorem to GRW models, Conway and Kochen mistakenly assume that the response of a particle (i.e. the outcome of a measurement) may depend only on the jumps which occurred in the backward light-cone of the spacetime point where the measurement occurs, since they regard the jumps as information which must fulfill FIN. But this cannot possibly be the right picture, even at a relativistic level, if the GRW model is to account for the nonlocal features of entangled quantum systems, which have been elucidated by Bell's work; we now discuss this issue in more detail.

\section{MODELS OF SPONTANEOUS WAVEFUNCTION COLLAPSE}

We briefly review some of the main features of the GRW model of spontaneous wavefunction collapse, which are relevant for the present discussion. We will first present the non relativistic 
GRW model [14] and then comment on possible relativistic generalizations.

\subsection{The GRW Model}

The starting point of the GRW model is that the wavefunction alone is the complete mathematical description of all physical phenomena, it represents the maximum knowledge one can have, in principle, about the state of a physical system, both microscopic and macroscopic. Since macroscopic objects are always well localized in space, while the Schrödinger equation allows for superpositions of different macroscopic states, one has to modify the standard quantum evolution in order to provide a consistent and unified description of micro- and macro-phenomena. This is done in the following way.

Let us consider a system of $N$ particles; let $\mathcal{H}$ be the Hilbert space associated to it and $H$ the standard quantum Hamiltonian of the system. The model is defined by the following postulates:

1. At random times, each particle experiences a sudden jump of the form:

$$
\psi_{t} \quad \longrightarrow \quad \frac{L_{n}(\mathbf{x}) \psi_{t}}{\left\|L_{n}(\mathbf{x}) \psi_{t}\right\|},
$$

where $\psi_{t}$ is the statevector of the whole system at time $t$, immediately prior to the jump process. $L_{n}(\mathbf{x})$ is a linear operator which is conventionally chosen equal to:

$$
L_{n}(\mathbf{x})=\sqrt[4]{\left(\frac{\alpha}{\pi}\right)^{3}} \exp \left[-\frac{\alpha}{2}\left(\mathbf{q}_{n}-\mathbf{x}\right)^{2}\right]
$$

where $\alpha$ is a new parameter of the model which sets the the width of the localization process, and $\mathbf{q}_{n}$ is the position operator associated to the $n$-th particle. The random variable $\mathbf{x}$ corresponds to the place where the jump occurs.

2. Between two consecutive jumps, the statevector evolves according to the standard Schrödinger equation.

3. The probability density for a jump taking place at the position $\mathbf{x}$ is given by:

$$
p_{n}(\mathbf{x}) \equiv\left\|L_{n}(\mathbf{x}) \psi_{t}\right\|^{2}
$$

the probability density for the different particles are independent.

4. Finally, it is assumed that the jumps are distributed in time according to a Poissonian process with frequency $\lambda$, which is the second new parameter of the model.

The standard numerical values for $\alpha$ and $\lambda$ are $^{4}$ :

$$
\lambda \sim 10^{-16} \mathrm{sec}^{-1} \quad \alpha \sim 10^{-10} \mathrm{~cm}^{-2},
$$

\footnotetext{
${ }^{4}$ Recently S.L. Adler proposed a radically different numerical value for the collapse rate $\lambda$; see ref. [15].
} 
which have been chosen in such a way to guarantee a very good agreement of GRW with standard quantum mechanics and, at the same time, to ensure an almost instantaneous localization of the wavefunction of classical macro-objects, thus suppressing the unwanted superpositions of differently located macro-states.

The evolution being stochastic, any initial state $\psi_{0}$ evolves in time into an ensemble of states $\left\{\psi_{t}(\omega)\right\}$, where $\omega$ labels the possible different ways the jumps might occur. The statistical operator $\rho_{t}$ associated to such an ensemble satisfy the following Lindblad-type equation:

$$
\frac{d}{d t} \rho_{t}=-\frac{i}{\hbar}\left[H, \rho_{t}\right]-\lambda \sum_{n=1}^{N}\left(\rho_{t}-\int d^{3} x L_{n}(\mathbf{x}) \rho_{t} L_{n}(\mathbf{x})\right) .
$$

The GRW model and similar models which have appeared in the literature have been extensively studied (see [16, 17] for a review of the subject); in particular, the following three important properties have been proved:

- At the microscopic level, quantum systems behave almost exactly as predicted by standard Quantum Mechanics, the differences between the predictions of the GRW model and of Quantum Mechanics being so tiny that they cannot be detected with present-day technology.

- At the macroscopic level, wavefunctions of macro-objects are almost always very well localized in space, so well localized that their centers of mass behave, for all practical purposes, like point-particles moving according to Newton's laws.

- In a measurement-like situation, e.g. of the von Neumann type, GRW reproduces - as a consequence of the modified dynamics - both the Born probability rule and the postulate of wave-packet reduction.

Accordingly, models of spontaneous wavefunction collapse provide a unified description of all physical phenomena, at least at the non-relativistic level, and a consistent solution to the measurement problem of Quantum Mechanics.

\subsection{Features of the GRW Model}

There are some important properties of the GRW model, which all non-relativistic collapse models share, and which are relevant for the subsequent discussion.

Non-linearity and stochasticity. The jump process is non-linear, since the probability of a jump taking place at $\mathbf{x}$ depends on the square norm of the statevector after the hitting. It is 
also intrinsically stochastic; the model assumes that Nature is fundamentally random; needless to say, such a property is important in order to recover quantum probabilities when measurement situations are taken into account, but also for other reasons which will be clear soon.

Non-locality. The model is manifestly non-local; let us take as an example the following entangled state of two particles $a$ and $b$ :

$$
\psi\left(\mathbf{x}_{a}, \mathbf{x}_{b}\right)=\frac{1}{\sqrt{2}}\left[\psi_{\Delta_{1}}\left(\mathbf{x}_{a}\right) \psi_{\Delta_{2}}\left(\mathbf{x}_{b}\right)+\psi_{\Delta_{3}}\left(\mathbf{x}_{a}\right) \psi_{\Delta_{4}}\left(\mathbf{x}_{b}\right)\right]
$$

where $\psi_{\Delta_{1}}(\mathbf{x})$ is a normalized wavefunction well localized within the region $\Delta_{1}$ of space, and similarly for the other three terms in $(11) ; \Delta_{1}, \Delta_{2}, \Delta_{3}$ and $\Delta_{4}$ label four regions which are arbitrarily far away from each other. Let us suppose that an experimenter $A$ decides to measure the position of particle $a$, while an experimenter $B$ decides to measure the position of particle $b$. The full initial state of the two particles plus the two apparata is:

$$
\psi_{\text {before }}\left(\mathbf{x}_{a}, \mathbf{x}_{b} ; \mathbf{y}_{\mathcal{A}}, \mathbf{y}_{\mathcal{B}}\right)=\frac{1}{\sqrt{2}}\left[\psi_{\Delta_{1}}\left(\mathbf{x}_{a}\right) \psi_{\Delta_{2}}\left(\mathbf{x}_{b}\right)+\psi_{\Delta_{3}}\left(\mathbf{x}_{a}\right) \psi_{\Delta_{4}}\left(\mathbf{x}_{b}\right)\right] \otimes \phi_{\text {Ready }}\left(\mathbf{y}_{\mathcal{A}}\right) \phi_{\text {Ready }}\left(\mathbf{y}_{\mathcal{B}}\right),
$$

where $\phi\left(\mathbf{y}_{\mathcal{A}}\right)$ and $\phi\left(\mathbf{y}_{\mathcal{A}}\right)$ denote the (localized) states of, let us say, the pointers of the two apparata, which initially are both in a state which is "ready" for the measurement.

Now, let us suppose that the position of particle $a$ is measured slightly before that of particle b. The dynamics of the GRW model tells that-because of the spontaneous jumps whose effect is amplified when a macro-object like a measuring apparatus enters into play - the final state of the pointer of the apparatus will be perfectly localized in space and will correspond either to the outcome $\mathcal{A}: \Delta_{1}$ or to the outcome $\mathcal{A}: \Delta_{3}$, each occurring with a probability almost identical to that given by the Born probability rule; let us suppose that the first possibility occurs. Then, the GRW dynamics implies that the initial state (12), after the first measurement, practically reduces to:

$$
\psi_{\text {after }}\left(\mathbf{x}_{a}, \mathbf{x}_{b} ; \mathbf{y}_{\mathcal{A}}, \mathbf{y}_{\mathcal{B}}\right)=\psi_{\Delta_{1}}\left(\mathbf{x}_{a}\right) \psi_{\Delta_{2}}\left(\mathbf{x}_{b}\right) \otimes \phi_{\mathcal{A}: \Delta_{1}}\left(\mathbf{y}_{\mathcal{A}}\right) \phi_{\text {Ready }}\left(\mathbf{y}_{\mathcal{B}}\right)
$$

We see that, because of the (local) jumps which occurred on the pointer of the measuring device used by $A$ to measure the position of particle $a$, also particle $b$ has been almost instantaneously localized in space, in this case within $\Delta_{2}$, no matter how distant $\Delta_{2}$ is from $\Delta_{1}$; in fact, as we see from state (13), a subsequent measurement of the position of particle $b$ will give (almost) certainly the outcome $\mathcal{B}: \Delta_{2}$. In a similar way, if the outcome of the first measurement had been $\mathcal{A}: \Delta_{3}$, then particle $b$ would have been immediately localized around $\Delta_{4}$, and this would be confirmed by any subsequent measurement of its position. 
Accordingly, there is a perfect and non-local correlation between the region where particle $a$ is located after a measurement and the region where particle $b$ is located by that same measurement done on its far away partner. Note, however, that the jumps acting on the pointer (and determining in this way the outcome of a measurement) are the consequence of a perfectly local interaction between the pointer and the stochastic background which enters in the dynamical evolution; it is only the entanglement between the two particles which renders the overall effect fundamentally non-local.

The crucial point to understand is that this non-local feature of the collapse mechanism is not a consequence of the fact that the GRW model is non-relativistic ${ }^{5}$; on the contrary, such a peculiar feature is necessary in order to reproduce the quantum correlations for EPR states like (11), which have been confirmed by all experiments. In other words, after the work of Bell and the experiments of Aspect which have shown that Nature is fundamentally non-local, any GRW model (whether relativistic or not) has to embody such a non-local behavior in order to reproduce quantum correlations.

No faster-than-light. One might wonder whether the non-local character of the jump process might be used to send faster-than-light signals, but in [12] it has been proven that this is not possible, and the physical reason is quite simple to understand: since jumps are intrinsically random, they cannot be controlled to implement faster-than-light communication, and as soon as one averages over all possible jumps, their non-local character vanishes.

Accordingly, like standard Quantum Mechanics, also the GRW model shares the "peaceful coexistence" between relativity and non-locality, which is one of the lessons we had lo learn from Bell's inequalities. Indeed, Bell himself has stated [19]: “... I am particularly struck by the fact that the [GRW] model is as Lorentz invariant as it could be in the nonrelativistic version. It takes away the ground of my fear that any exact formulation of quantum mechanics must conflict with fundamental Lorentz invariance".

The formal aspects of this nice feature of the theory consists in the fact that GRW violates Bell's locality condition by violating outcome independence, just as standard nonrelativistic quantum mechanics does. Before proceeding it is useful to recall that Bell's locality assumption has been proved $[20,21]$ to be equivalent to the conjunction of the two logically independent conditions, parameter independence and outcome independence. To clarify the matter let us fix our notation.

\footnotetext{
${ }^{5}$ Indeed, it would be very easy to devise a local jump process, even for entangled states, which in any case would lead to a conflict with quantum mechanical predictions.
} 
We will denote by $\lambda$ all parameters (which may include the quantum mechanical statevector or even to reduce to it alone) that specify completely the state of an individual physical system. For simplicity we will refer to a standard EPR-Bohm setup and we will denote by $p_{\lambda}^{A B}(x, y ; n, m)$ the joint probability of getting the outcome $x$ in a measurement at $A$ and $y$ in a measurement at $B$. Obviously we assume that the experimenters at $A$ and $B$ can make a free-will choice of the directions $n$ and $m$ along which they will perform their measurements. They can also choose not to perform the measurement. Bell's locality assumption can be expressed as

$$
p_{\lambda}^{A B}(x, y ; n, m)=p_{\lambda}^{A}(x ; n, *) p_{\lambda}^{B}(y ; *, m)
$$

where the symbol $*$ appearing on the r.h.s. denotes that the corresponding measurement is not performed. As already anticipated, the above condition has been proved to be equivalent to the conjunction of the two following conditions:

$$
p_{\lambda}^{A}(x ; n, m)=p_{\lambda}^{A}(x ; n, *) ; \quad p_{\lambda}^{B}(y ; n, m)=p_{\lambda}^{B}(y ; *, m)
$$

and

$$
p_{\lambda}^{A B}(x, y ; n, m)=p_{\lambda}^{A}(x ; n, m) p_{\lambda}^{B}(y ; n, m),
$$

where we have denoted, e.g., by the symbol $p_{\lambda}^{A}(x ; n, m)$ the probability of getting, for the given settings $n, m$, the outcome $x$ at A. The first conditions express Parameter independence, i.e., the requirement that the probability of getting an outcome at $A(B)$ is independent of the setting chosen at $B(A)$, while the last conditions (Outcome independence) expresses the requirement that the probability of an outcome at one wing does not depend on the outcome obtained at the other wing.

We are now in the conditions of discussing briefly this point with reference to the twined state of total spin 0 of Conway and Kochen:

$$
\left|\phi_{\text {singlet }}\right\rangle=\frac{1}{\sqrt{3}}\left[\left|S_{a: n}=+1\right\rangle\left|S_{b: n}=-1\right\rangle+\left|S_{a: n}=-1\right\rangle\left|S_{b: n}=+1\right\rangle-\left|S_{a: n}=0\right\rangle\left|S_{a: n}=0\right\rangle\right],
$$

the states $\left|S_{a: n}=+1\right\rangle,\left|S_{a: n}=0\right\rangle,\left|S_{a: n}=-1\right\rangle$ and the similar ones for particle $b$ being the eigenstates belonging to the indicated eigenvalues of the spin component along an arbitrary direction $n$. Let us now consider particle $b$. If particle $a$ is not subjected to any measurement, the probabilities of the two outcomes for $S_{b: n}^{2}$ are ${ }^{6}$ :

$$
P\left(S_{b: n}^{2}=1\right)=\frac{2}{3}, \quad P\left(S_{b: n}^{2}=0\right)=\frac{1}{3} .
$$

\footnotetext{
${ }^{6}$ As already remarked the GRW model gives practically the same predictions and has the same effects as standard quantum mechanics with the wave packet reduction postulate.
} 
Suppose now that particle $a$ has been subjected to a measurement of $S_{a: n}^{2}$ : the outcome is either 1 or 0 ; then $P\left(S_{b: n}^{2}=1\right)=1$ and $P\left(S_{b: n}^{2}=0\right)=0$ or viceversa, according to the outcome which has been obtained. Such probabilities differ from those of no measurement on particle $a$. This shows that the theory exhibits Outcome Dependence. However, if one performs a non-selective measurement on particle $a$, one gets the outcome 1 with probability $\frac{2}{3}$ and the outcome 0 with probability $\frac{1}{3}$. Since reduction takes place to the states $\frac{1}{\sqrt{2}}\left[\left|S_{a: n}=+1\right\rangle\left|S_{b: n}=-1\right\rangle+\left|S_{a: n}=-1\right\rangle\left|S_{b: n}=+1\right\rangle\right]$ and $\left|S_{a: n}=0\right\rangle\left|S_{b: n}=0\right\rangle$, respectively, the probabilities of the two outcomes for a measurement on particle $b$ coincide precisely with those of the case of no measurement. The model then does not exhibit Parameter Dependence. Concluding: the GRW model violates locality by violating only outcome independence and, as well known, since this feature of the theory does not forbid having a relativistic quantum theory, it therefore does not forbid relativistic dynamical reduction processes. Stochastic Galilean invariance. When dealing with a theory like the GRW model which contemplates a stochastic evolution, one has to be careful in specifying the precise meaning of the theory being invariant under the transformations of a symmetry group. In the nonrelativistic case this group is naturally identified with the Galilei group, which, however, in the present case must be restricted to the so called Galilei semigroup $(G S)$ which contains only forward time translations, since the theory has a built in arrow of time.

Let us then consider an observer $O$ who prepares a system in a state $\left|\Psi_{0}^{O}\right\rangle$ and lets it evolve, under the combined effect of the purely Hamiltonian evolution and the random localization processes up to time $t$, getting the state $\left|\Psi_{t}^{O}(\omega)\right\rangle$. Here $\omega$ specifies the precise localizations processes which have taken place in the interval $(0, t)$. Obviously, since the localizations are random processes, the state $\left|\Psi_{t}^{O}(\omega)\right\rangle$ has a certain probability $P_{\Psi_{0}^{O}}(\omega)$, which depends on the initial state and the localizations which took place, of being the state describing the situation at time $t$.

Let us consider now another observer $O^{\prime}$ which is related to $O$ by a transformation $g \in G S$. We know how the states associated by $O$ and $O^{\prime}$ are related: $O^{\prime}$ associates to the system states which are obtained from those of $O$ by the unitary operator $U_{g}$ implementing the transformation $g \in G S$ on the Hilbert space of the system.

We can now make precise the notion of stochastic invariance. The theory is invariant for the transformations of the Galilei semigroup if its dynamics implies that the transformed initial state $U_{g}\left|\Psi_{0}^{O}(\omega)\right\rangle$ has precisely the probability $P_{\Psi_{0}^{O}}(\omega)$ (i.e. the one characterizing the evolution for $O$ ) of evolving into the transformed state $U_{g}\left|\Psi_{t}^{O}(\omega)\right\rangle$. In ref. [18] this has been proved to be authomatically true within GRW if the hamiltonian governing the pure Schrödinger evolution is invariant for the considered transformation, in the usual sense of standard quantum mechanics. In 
simple words, the GRW model is stochastically invariant under the transformations of the Galilei semigroup $^{7}$.

\subsection{Relativistic GRW Models}

In the past years a great deal of work has been done in order to formulate a relativistic version of the GRW model, and some interesting results have been achieved, even though no final, fully satisfactory model is available yet. To be coincise, it suffices here to make only a few general remarks, all of which should be clear from the previous discussion of the GRW model:

- Any relativistic extension of the GRW model must be non-linear and stochastic in order to provide a solution to the measurement problem of Quantum Mechanics.

- Such an extension must be also non-local, if it aims at reproducing quantum correlations for EPR types of experiments, in particular when they are performed at space-like separated regions; in one way or another, the jump process, even though it is triggered locally, must "propagate" practically instantaneously. As a consequence, the (stochastic) equation for the evolution of the statevector $\left|\psi_{t}\right\rangle$ must be highly non-local.

- At the same time, the extension must satisfy the no faster-than-light constraint, in order to be compatible with special relativity. From the previous analysis the strategy one has to adopt in order to achieve such a result should be clear: due to stochasticity, only the average effect of the jump processes, not the single realizations of the jumps, can be controlled; accordingly, the statistical properties of the jump mechanism must be chosen in such a way that, when the average over all possible realizations is taken, no non-local feature can be exploited to send signals at a speed greater than the speed of light. Stated in more mathematical terms, while the equation for $\left|\psi_{t}\right\rangle$ must be non-local, the equation for the statistical operator $\rho_{t}$ must be local in the sense that no measurement done in any region of

\footnotetext{
${ }^{7}$ In a recent nice paper [22], the authors have appropriately stressed the necessity of enriching the pure formal structure of a theory by what they have denoted as its Primitive Ontology (PO) and they have mentioned that for the GRW model one can at least choose two PO's, which they call the Flashes Ontology and the Mass Density Ontology, respectively (we refer the interested reader to the paper). Here we remark simply that the invariance property we have just discussed implies that the GRW theory can be claimed to be invariant according to the definition they have used for this purpose: To say that a theory has a given symmetry is to say that the possible histories of the PO, those which are allowed by the theory, when transformed according to the symmetry, will again be possible histories for the theory, and the probability distribution on the histories supplied by the theory, when transformed, will again be a possible probability distribution for the theory.
} 
space can in any way alter instantaneously the statistical distribution of the outcomes with respect to measurements done at space-like separated regions.

- Finally, the extension will not be Lorentz invariant in the ordinary sense, but in the stochastic sense previously discussed in connection with the GRW model.

Of course, the major difficulty is to put all these ideas into a consistent model, with a working equation; as already remarked, no fully satisfactory model has been developed so far, but we want to draw attention to ref. [23], where a general framework for relativistic reduction models is presented. In the quoted paper the analysis is performed with reference to a toy model which does not assume a specific dynamics for the reduction mechanism (this is where the difficulty in making the GRW model relativistically invariant lies, and the reason why this problem is still open), but it allows one to conclude that there is no reason of principle forbidding the relativistic reduction program. Within this framework, following previous ideas of Aharonov and Albert on relativistic formulations of the postulate of wave-packet reduction [24, 25, 26], the collapse mechanism is supposed to occur instantaneously along all spacelike hypersurfaces crossing the center of the jump process; in spite of this superluminal effect, the whole picture is perfectly Lorentz invariant (in the stochastic sense), it agrees with quantum mechanical predictions, it does not lead to any contradiction, e.g. it does not allow faster-than-light signalling and, moreover, different inertial observers always agree on the outcomes of experiments. Another model has been recently proposed by R. Tumulka [27]: it is based on the multi-time formalism with $N$ Dirac equations, one per particle, and up to now it works only for non-interacting particles. The open question is whether it can be consistently generalized to include also interactions.

\section{THE CONWAY-KOCHEN ARGUMENT AND RELATIVISTIC GRW}

From the previous analysis it should be clear why the argument by Conway and Kochen does not apply to possible relativistic extensions of the GRW model: since such an extension must necessarily be non-local in order to reproduce quantum correlations, it must violate FIN, and the whole argument breaks down. We now repeat again the argument in the light of a GRW-like description of quantum phenomena, to see where and how non-locality enters into play, and also to point out a mistake made by Conway and Kochen in discussing the collapse process. Let us consider again the singlet state (17) of total spin 0 for two spin 1 particles, and let us assume that the two observes $A$ and $B$ perform the two measurements of the square of the spin in two space-like 
separated regions of spacetime; the complete state of the two particles plus the two apparata is:

$$
\left|\psi_{\text {before }}\right\rangle=\left|\phi_{\text {singlet }}\right\rangle \otimes \phi_{\text {Ready }}\left(\mathbf{y}_{\mathcal{A}}\right) \phi_{\text {Ready }}\left(\mathbf{y}_{\mathcal{B}}\right)
$$

Needless to say, the FREE axiom must be true, thus $B$ is free to measure along any triple of directions. Let us suppose that, with respect to a given reference frame, he is the first who does the measurement; he chooses the triple $n, j, k$ and obtains e.g. the result:

$$
S_{b: n}^{2}(j, k ; \beta)=1, \quad S_{b: j}^{2}(n, k ; \beta)=1, \quad S_{b: k}^{2}(n, j ; \beta)=0
$$

Now the crucial point comes. If we require the putative GRW model to fulfill the TWIN axiom, i.e. if we require (and we want to require it) the model to agree with quantum mechanical predictions, then we must admit that, as a consequence of the measurement made by $B$ on particle $b$ - i.e. as a consequence of the jumps acting on the device used by $B$ in such a way to determine the required outcome - the whole state of the two particles must change almost immediately (and in a non-local manner) from the state (19) to the new state:

$$
\left|\psi_{\text {after }}\right\rangle=\left|S_{a: k}=0\right\rangle\left|S_{b: k}=0\right\rangle \otimes \phi_{\text {Ready }}\left(\mathbf{y}_{\mathcal{A}}\right) \phi_{\mathcal{B}: S_{b: k}=0}\left(\mathbf{y}_{\mathcal{B}}\right)
$$

only in this way we can be sure that, if $A$ decides to measure the square of the spin of particle $a$ along the same three directions $n, j, k$ chosen by $B$, he will certainly get the same results which $B$ obtained. We stress it again: a non-local and almost instantaneous collapse from state (19) to (21) is necessary in order for the putative model to account for the quantum correlations involving entangled states.

But now we see that the state of particle $a$ has changed because of something which happened outside its backward light-cone (the jumps acting on the device used by $B$ at a space-like separated distance), and this change will affect the response of $a$ to spin measurements. Moreover, the new state of particle $a$ depends on the choice of directions chosen by $B$ to measure the square of the spin of particle $b$. Because of this non-local dependence, the argument of Conway and Kochen does not hold anymore.

In their criticism of relativistic GRW models, Conway and Kochen mistakenly assume that the jump processes somehow have a local character, i.e. that only the jumps which occurred in the backward light-cone can affect the behavior of a particle. But, as we have seen this is not the right picture: the reduction mechanism, even though it is triggered by a perfectly local particleapparatus interaction, must have a well-defined non-local character such that the outcome of the measurement which $A$ performs on particle a does - indeed, it must - depend on the outcome of the 
measurement performed by $B$ on $b$ (or vice-versa), in particular on the choice of directions made by $B$, even when the two measurements occur at space-like separated regions. Accordingly, the putative model deliberately violates FIN, because the jumps (which Conway an Kochen regard as part of information) propagate instantaneously. But this does not necessarily generate a conflict with special relativity since, as previously discussed, such jumps must be such that they cannot be used to send signals at a speed greater than the speed of light ${ }^{8}$.

Of course, one might wonder how it is possible to reconcile such a highly non-local behavior of the jump mechanism with special relativity; as we pointed out in Sec. II, this is still an open problem, a tentative solution to which has been given in [23] and [27].

To conclude, because relativistic extensions of the GRW model which reproduce quantum mechanical correlations must be non-local in one way or another, the Conway and Kochen argument does not apply to them: the problem of finding a fully convincing relativistic model of spontaneous wavefunction collapse remains open. Of course it is a difficult problem, but there is no reason of principle which makes it impossible.

\section{ACKNOWLEDGEMENTS}

We wish to thank S.L. Adler, D. Deckert, D. Dürr, S. Goldstein, R. Tumulka and N. Zanghì for many useful comments.

[1] J. Conway and S. Kochen, Found. Phys. (2006), to appear. ArXiv: quant-ph/0604079v1 (11 Apr 2006).

[2] S. Kochen and E. Specker, J. Math. Mech. 17, 59 (1967).

[3] P. Heywood and M.L.G. Redhead, Found. Phys. 13, 481 (1983).

[4] A. Stairs, Phil. Sci. 50, 578 (1983).

[5] H.R. Brown and G. Svetlichny, Found. Phys. 20, 1379 (1990).

[6] A. Elby, Found. Phys. 20, 1389 (1990).

[7] A. Aspect, P. Grangier and G. Roger, Phys. Rev. Lett. 49, 91 (1982);

[8] A. Aspect, J. Dalibard and G. Roger, Phys. Rev. Lett. 49, 1804 (1982).

[9] P.H. Eberhard, Nuovo Cimento 46 B, 392 (1978).

[10] G.C, Ghirardi, A. Rimini and T. Weber, Lett. Nuovo Cimento 27, 293 (1980).

\footnotetext{
${ }^{8}$ Contrary to Conway and Kochen, we think that the jump processes should not be regarded as information, whatever this word precisely means. The reason is that they cannot be known ahead of time, they cannot be controlled, and they cannot be used to convey other information ..., i.e., they do not behave as what is typical of information.
} 
[11] A. Shimony, Intern. Philos. Quarterly 18, 3 (1978).

[12] J. Butterfield, G. Fleming, G.C. Ghirardi and R. Grassi, Int. J. Theor. Phys. 32, 2287 (1993).

[13] D. Hemmick, Hidden Variables and Nonlocality in Quantum Mechanics (Ph.D. dissertation, 1996).

[14] G.C. Ghirardi, A. Rimini and T. Weber, Phys. Rev. D 34, 470 (1986).

[15] S.L. Adler, J. Phys. A, to appear. ArXhiv: quant-ph/0605072v8 (23 Aug 2006).

[16] A. Bassi and G.C. Ghirardi, Phys. Rept. 379, 257 (2003).

[17] P. Pearle, in Open systems and measurement in relativistic quantum theory, Lecture Notes in Physics 526, H.-P. Breuer and F. Petruccione eds. (Springer-Verlag, Berlin, 1999).

[18] G.C. Ghirardi, R. Grassi and P. Pearle, Found. Phys. 20, 1271 (1990).

[19] J.S. Bell, Speakable and unspeakable in Quanum Mechanics (Cambridge University Press, Cambridge, 1987).

[20] J. Jarret, Nous 18, 569 (1984).

[21] P. Suppes and M. Zanotti, in Logic and Probability in Quantum Mechanics, P. Suppes ed. (Reidel, Dordrecht, 1976).

[22] V. Allori, S. Goldstein, R. Tumulka and N. Zanghì, ArXiv quant-ph/0603027v2 (26 Jun 2006).

[23] G.C. Ghirardi, Found. Phys. 30, 1337 (2000).

[24] Y. Aharonov and D.Z. Albert, Phys. Rev. D 24, 359 (1981).

[25] Y. Aharonov and D.Z. Albert, Phys. Rev. D 21, 3316 (1980).

[26] Y. Aharonov and D.Z. Albert, Phys. Rev. D 29, 228 (1984).

[27] R. Tumulka, arXiv quant-ph/0406094v1 (14 Jun 2004). 